\title{
A 90nm, Low Power VCO with Reduced KVCO and Sub-band Spacing Variation
}

\author{
Diarmuid Collins, Aidan Keady, Grzegorz Szczepkowski, Ronan Farrell \\ IMWS, Dept. of Electronic Engineering, NUI Maynooth, Ireland \\ MCCI, Tyndall National Institute, Cork, Ireland \\ Email: dcollins@eeng.nuim.ie
}

\begin{abstract}
In this paper we present the design of a low power VCO with reduced variations in VCO gain $\left(K_{V C O}\right)$ and subband spacing resolution $\left(f_{r e s}\right)$. The proposed VCO is designed using a 90nm CMOS process to cover a tuning range of $23 \%$. Variations in $K_{V C O}$ and $f_{\text {res }}$ are reduced by factors of 6 and 17 respectively over a conventional sub-banded VCO, designed using the same process, to meet the same tuning range. This makes the proposed VCO more suited to stable PLL operation with its reduced $\mathbf{K}_{V C O}$ requirements resulting in an improvement in phase noise performance over the conventional VCO by $2 \mathrm{~dB}$. Due to the reduced loading on the VCO tank achieved by the presented design, power consumption is kept extremely low at $850 \mu W$ from a $1 \mathrm{~V}$ supply.
\end{abstract}

\section{INTRODUCTION}

Phase-tocked loops (PLLs) are important building blocks in modern communication systems. At the heart of the PLL is a voltage-controlled oscillator (VCO), responsible for generating the output frequency of the PLL. In order to account for process, voltage and temperature (PVT) variation, and to support multi-standard operation, the VCO is required to operate over a sufficiently large frequency range. This requirement, together with the reduced supply voltages that accompany modern low power nanoscale technologies, imply the design of a VCO with a large tuning constant $\left(K_{V C O}-\right.$ frequency change per volt). A large $K_{V C O}$ is however highly undesirable in terms of optimum phase noise performance as it translates any noise on the VCO control line to frequency modulation of the output signal. This leads to increased jitter of the output signal, which can be particularly problematic for PLLs operating as frequency synthesizers [1].

This issue is typically addressed by dividing the tuning range into discrete smaller bands (sub-bands), such that a wide tuning range can be realised with a small $K_{V C O}$ for each subband [2]. Illustrated in Fig. 1 is a VCO tuning curve which has been divided into $n$ discrete sub-bands to achieve the same original tuning range, but with a smaller $K_{V C O}$.

The illustration of Fig. 1 is not realistic however, as in practice sub-band spacing resolution $\left(f_{r e s}\right)$ and $K_{V C O}$ are not constant. Instead they reduce over sub-bands, resulting in a reduction in the frequency range covered by the VCO. This leads to excessive $K_{V C O}$ values required to meet the specified tuning range which will degrade the phase noise performance of the VCO. In addition, $\triangle K_{V C O}$ is very severe on PLL operation as PLL loop bandwidth is proportional
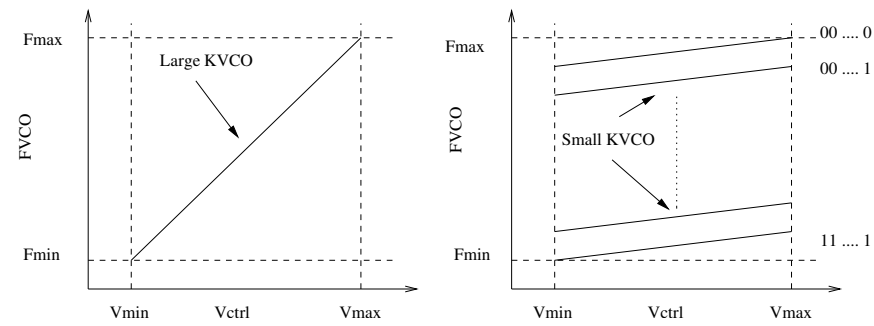

Fig. 1. Sub-banding of single VCO tuning curve

to $K_{V C O}$. Therefore, in the absence of additional calibration, loop bandwidth will vary with $\Delta K_{V C O}$ hence affecting the noise performance and loop stability of the PLL itself. Although a reduction in $\Delta K_{V C O}$ alone will improve PLL noise performance and loop stability, it should be accompanied by a corresponding reduction in $f_{\text {res }}$ variation $\left(\Delta f_{\text {res }}\right)$ to optimise the potential tuning range and achieve a more PVT robust solution. Therefore to address this issue, we present the novel design of a low power, sub-banded VCO with reduced $\Delta K_{V C O}$ and $\Delta f_{\text {res }}$.

The paper is organised as follows. Section II details the problem of $\Delta K_{V C O}$ and $\Delta f_{\text {res }}$. Related work is reviewed in Section III. The proposed technique and circuit implementation are presented in sections IV and V. Experimental results are then presented in section VI with a conclusion given in section VII.

\section{SUB-BANDED VCOS}

Dividing the tuning range into $n$ sub-bands is conventionally achieved using a switched capacitor array which consists of $n-1$ equi-valued (or binary weighted) switchable capacitors, selectable via a digital word. The switching in(out) of one capacitor adds(subtracts) from the total tank capacitance, forcing VCO operation to move down(up) one sub-band respectively.

The reduction in $K_{V C O}$ occurs because the percentage change in capacitance, caused by the varactor, reduces as more capacitance is added to the tank. The reduction in $f_{\text {res }}$ then occurs because the percentage change in capacitance, caused by switching in a capacitor, reduces as more capacitance is added to the tank. As a result both $K_{V C O}$ and $f_{\text {res }}$ reduce as more capacitors are switched in.

In [3] it was shown that band to band variation 
reduces $K_{V C O}$ by the following factor:

$$
\Delta K_{V C O}=C \sqrt{C}
$$

where $C$ represents a change in capacitance (for example: from switching in the entire switched capacitor array).

This changes $f_{\text {res }}$ by the same factor which, as shown in (2), preserves a constant percentage overlap between bands:

$$
\Delta O V \propto \frac{\Delta f_{\text {res }}}{\Delta K_{V C O}}
$$

where $\triangle O V$ represents the variation in percentage overlap between bands.

As a result, the overall frequency range covered by the VCO also reduces by the same factor leading to a reduction in the tuning range as follows:

$$
T R_{\text {reduced }}=\frac{\left(\frac{2 \Delta F}{C \sqrt{C}}\right)}{2 F_{\max }-\left(\frac{\Delta F}{C \sqrt{C}}\right)} \cdot 100
$$

where the reduced tuning range $(\%)$, frequency range and maximum frequency of the VCO are represented by $T R_{\text {reduced }}$, $\Delta F$ and $F_{\max }$ respectively.

As seen from (3), the original tuning range can simply be restored by increasing $\Delta F$ by $C \sqrt{C}$ which is achieved in practice by increasing the average $K_{V C O}$ by the same factor. This however is highly undesirable because, as explained in section I, any increase in $K_{V C O}$ leads to an increase in noise translation from VCO control line to the output signal.

\section{RELATED WORK}

Some methods have been proposed to reduce $\Delta K_{V C O}$ and $\Delta f_{\text {res }}$. In [4] the use of an additional serial $L C$-tank with variable inductor configuration is proposed to reduce $\Delta K_{V C O}$. Due to the additional $L C$-tank this solution consumes large power and die area. In [5] the use of additional varactors to reduce the $\triangle K_{V C O}$ of groups of sub-bands is proposed. This solution however requires an additional complex biasing scheme to reduce $\triangle K_{V C O}$ down to a specified level. In addition, both solutions do not address $\Delta f_{\text {res }}$. If $\Delta K_{V C O}$ is reduced then, as shown in (2), $\Delta f_{\text {res }}$ should also be reduced by the same amount to preserve a constant overlap between bands. This achieves a more PVT robust VCO which optimise's its dynamic tuning range while avoiding frequency dead-zones at extreme corners. In [6], [7], the use of an additional varactor array in conjunction with the conventional switched capacitor array is proposed to achieve simultaneous reductions in both $\Delta K_{V C O}$ and $\Delta f_{\text {res }}$. In [6] unused varactors in the array are simply switched out whilst those in [7] are connected to a fixed potential. Due to the additional array, the quality factor of the tank $\left(Q_{\text {tank }}\right)$ is degraded over conventional approaches, leading to degraded phase noise performance and increased power consumption.

\section{Proposed Technique}

The proposed technique simultaneously reduces both $\Delta K_{V C O}$ and $\Delta f_{r e s}$. Unlike [6], [7], this is achieved using one single array resulting in reduced loading of $Q_{\text {tank }}$. The array consists of $n-1$ switchable varactor branches to split the specified tuning range into $n$ sub-bands, as shown in Fig. 2. Each varactor branch is selectable via digital control signals which select(de-select) the branches by switching them in(out) respectively. De-selecting varactor branches by completely switching them out offers a more attractive solution over fixing them to a fixed potential [7], as it further reduces loading of $Q_{\text {tank }}$ for the higher frequency bands, where the majority of the branches will be switched out.

\section{A. Design Procedure}

Each varactor branch is sized according to its required $C_{\max }$ and $C_{\min }$ values necessary to achieve the specified $K_{V C O}$ and $f_{\text {res }}$. The procedure for calculating these values is as follows:

1) Calculate $C_{\min }$ for the sub-band of interest $\left(C_{\min _{n}}\right)$ :

$$
C_{\text {min }_{n}}=\frac{1}{L\left(2 \pi f_{\max _{n}}\right)^{2}}
$$

where $f_{\max _{n}}$ is the maximum frequency of the subband, taking into account the specified $f_{\text {res }}$. This is calculated as follows:

$$
\begin{aligned}
f_{\max _{n}} & =f_{\max _{n}-1}-f_{\text {res }} \\
& =f_{\max _{n}-1}-\left(\Delta f_{n-1}(1-O V)\right)
\end{aligned}
$$

where the maximum frequency and frequency range covered by the previous sub-band are $f_{\text {max }_{n}-1}$ and $\Delta f_{n-1}$ respectively.

2) Calculate the change in $C_{\min }$ between the sub-band of interest and the previous one $\left(\Delta C_{\min _{n} \rightarrow{ }_{n}-1}\right)$ :

$$
\Delta C_{\min _{n} \rightarrow{ }_{n}-1}=C_{\min _{n}}-C_{\min _{n}-1}
$$

where $C_{m_{i n}-1}$ is the minimum capacitance of the previous sub-band.

3) Calculate $C_{\max }$ for the sub-band of interest $\left(C_{\max _{n}}\right)$ :

$$
C_{\text {max }_{n}}=\frac{1}{L\left(2 \pi f_{\text {min }_{n}}\right)^{2}}
$$

where $f_{\text {min }_{n}}$ is the minimum frequency of the subband, taking into account the specified $K_{V C O}$. This is calculated as follows:

$$
f_{\text {min }_{n}}=f_{\text {max }_{n}}-\left(K_{V C O} V_{\text {tune }}\right)
$$

where $V_{\text {tune }}$ is the tuning voltage range.

4) Calculate the change in $C_{\max }$ between the sub-band of interest and the previous one $\left(\Delta C_{\max _{n} \rightarrow{ }_{n}-1}\right)$ :

$$
\Delta C_{\max _{n} \rightarrow_{n}-1}=C_{\max _{n}}-C_{\max _{n}-1}
$$

where $C_{\text {maxn }_{n}-1}$ is the maximum capacitance of the previous sub-band.

Equations (6) and (9) give the required $C_{\min }$ and $C_{\max }$ values respectively for the sub-band of interest. Once obtained, the relevant varactor branch is sized to achieve both values and inserted into the array. Repeating this procedure $n-1$ times thus completes the array. 


\section{Circuit Description}

To verify the proposed technique a low power, sub-banded VCO with center operating frequency of $4.8 \mathrm{GHz}$ was designed for a $1 \mathrm{P} 9 \mathrm{M} 90 \mathrm{~nm}$ CMOS process. The tuning range of the circuit is divided into 16 sub-bands with reduced $\Delta K_{V C O}$ and $\Delta f_{\text {res }}$. The schematic of the VCO is shown in Fig. 2.

The LC-tank consists of a differential spiral inductor $(L)$ and $\mathrm{p}-\mathrm{n}$ junction diode varactors $\left(D_{1}, D_{2}\right)$. A differential spiral inductor is used due to its higher quality factor $(Q)$ and lower area requirements over its single ended counterpart [8], which measured an inductance of $4 \mathrm{nH}$ and $Q$ of 16 at $4.8 \mathrm{GHz}$. $\mathrm{P}$-type diode varactors are used due to their higher $\mathrm{Q}-\mathrm{values}$ and linearity over the alternative N-type MOSFET varactors available for the process used. Anodes of both varactors are clamped to Vss through resistors $(R)$ to ensure no forward biasing, requiring them to be de-coupled from the tank using metal-insulator-metal capacitors $\left(C_{s}\right)$. This offers the additional advantage of further improving varactor linearity [9], thereby reducing AM-FM up-conversion of flicker noise from the bias source [10].

Flicker noise from the bias source can be completely eliminated by removing the bias source [11]. However this causes the amplitude of the oscillator signal to become more susceptible to PVT variations. Therefore to achieve a potentially more PVT robust circuit, a bias source is used which employs p-type MOSFETs $(P 1, P 2)$ due to their lower flicker noise. Negative resistance necessary for oscillation is then generated by the cross-coupled NMOS transistor pair $(N 1, N 2)$.
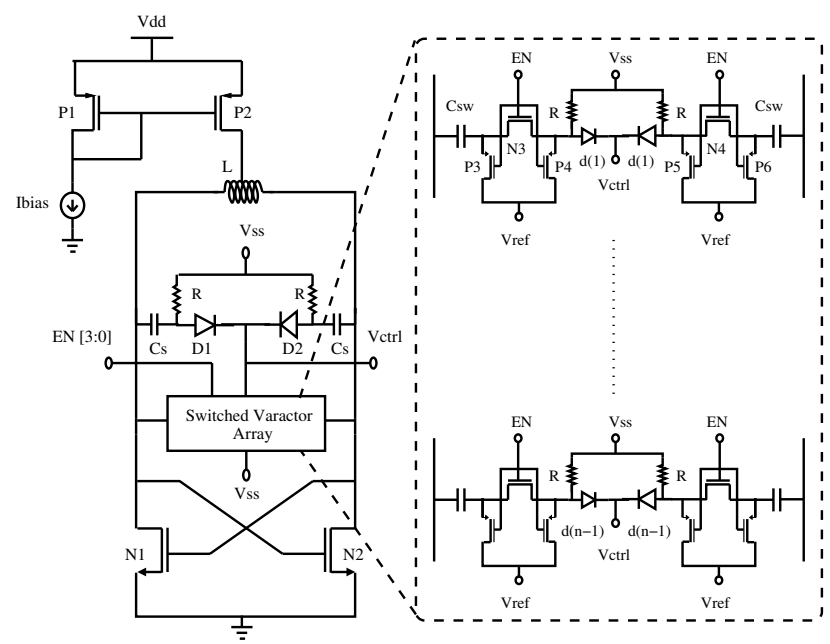

Fig. 2. Circuit diagram of the proposed VCO

The switched varactor array consists of 15 branches, also illustrated in Fig. 2. An identical varactor configuration that was used in the LC-tank is employed, where NMOS transistors $(N 3, N 4)$ are used to switch the varactors in(out) of the bank. These switches are made wide to reduce their series resistance which loads $Q_{\text {tank }}$ when $E N=1$. Loading of $Q_{\text {tank }}$ is further reduced by PMOS transistors $(P 3-P 6)$, which maintain the switches completely off when $E N=0$ [12].

\section{EXPERIMENTAL RESULTS}

The proposed VCO was designed for a 1P9M 90nm CMOS process. To demonstrate the achieved reductions in $\Delta K_{V C O}$ and $\Delta f_{\text {res }}$, the proposed VCO was compared with a conventional VCO designed using the same process, to meet the same tuning range. The resulting tuning characteristics of both VCOs are shown in Fig. 3, with a performance comparison of both circuits presented in Table I.

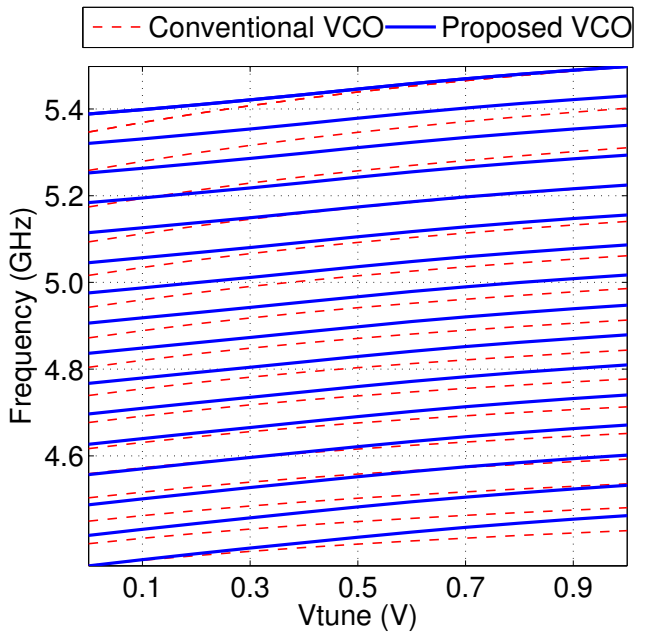

Fig. 3. Simulated tuning characteristics of the VCOs

TABLE I

PERFORMANCE SUMMARY COMPARISON

\begin{tabular}{|c|c|c|}
\hline & Conventional VCO & Proposed VCO \\
\hline Technology & \multicolumn{2}{|c|}{ 90nm CMOS } \\
\hline Power Supply & \multicolumn{2}{|c|}{$1 \mathrm{~V}$} \\
\hline$\Delta F$ & $(4.35 \mathrm{GHz}-5.5 \mathrm{GHz})$ \\
\hline TR & \multicolumn{2}{|c|}{$23 \%$} \\
\hline$\Delta K_{V C O}$ & $\pm 30 \%$ & $\pm 4.6 \%$ \\
\hline$\Delta f_{\text {res }}$ & $\pm 30 \%$ & $\pm 1.7 \%$ \\
\hline OV & $40 \%$ & $\approx 40 \%$ \\
\hline Power Consumption & $900 \mu \mathrm{W}$ & $850 \mu \mathrm{W}$ \\
\hline
\end{tabular}

Table I clearly shows the reductions achieved in $\Delta K_{V C O}$ and $\Delta f_{r e s}$ by factors of 6 and 17 respectively over the conventional VCO. This is shown in further detail in Figs. 4 and 5 which plot $K_{V C O}$ and $f_{\text {res }}$ respectively over all sub-bands for both VCOs.

As explained in section II and shown in Fig. 3, designing with reduced $\Delta K_{V C O}$ and $\Delta f_{\text {res }}$ enables the specified tuning range to be covered with lower $K_{V C O}$ values for the upper frequency bands, hence improving the phase noise performance of these bands. This improvement is shown in Fig. 6 which plots the simulated phase noise of the top frequency band for both VCOs (i.e. worst case scenarios). Measurements taken at $1 \mathrm{MHz}$ offset from the carrier frequency $\left(f_{o s c}=5.45 \mathrm{GHz}\right)$ show the proposed VCO to achieve a phase noise improvement of $2 \mathrm{~dB}$. This improvement is slightly larger than predicted due 

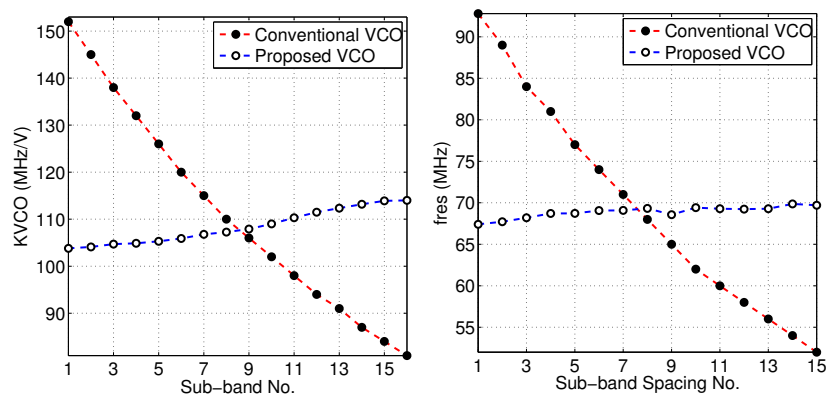

Fig. 4. $K_{V C O}$ over sub-bands Fig. $5 . f_{r e s}$ over sub-band spacings

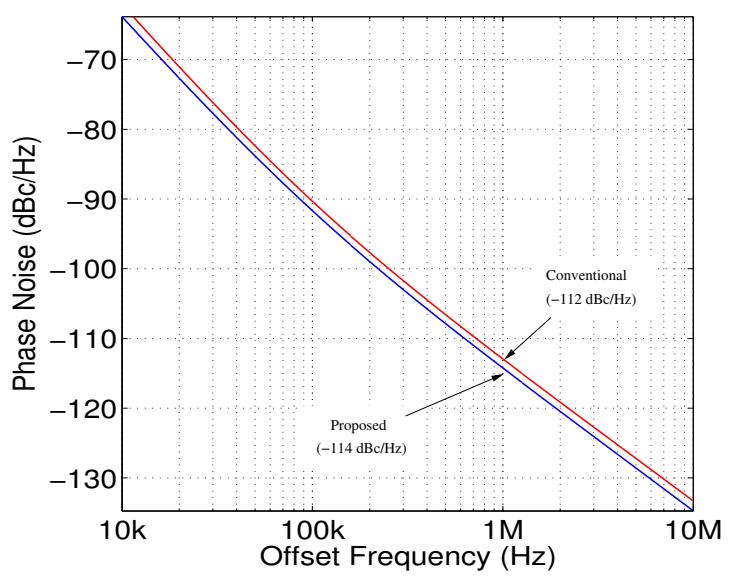

Fig. 6. Simulated Phase Noise of VCOs

to $K_{V C O}$ reduction alone, as the lower $K_{V C O}$ requirements for the proposed VCO will increase the $L / C$ ratio of its tank. This leads to an improved $Q_{\text {tank }}$ [8] hence offering a further improvement in phase noise.

Table I also shows the low power requirements of the proposed technique. This is due to the reduced loading of $Q_{\text {tank }}$ as a result of using one single array consisting of varactor branches which can be completely switched out when not in use. Power consumption is slightly less than for the conventional VCO (due to the higher $L / C$ tank ratio [8]) and is significantly less than for previously reported techniques concerned with reducing $\Delta K_{V C O}$ and $\Delta f_{\text {res }}$. This is shown in Table II which displays the overall performance of the proposed technique against such previously reported publications.

\section{CONCLUSION}

This paper presented a novel design of low power, subbanded VCO exhibiting reduced $\Delta K_{V C O}$ and $\Delta f_{\text {res }}$. The design was verified for a 90nm CMOS process and achieves $\Delta K_{V C O}$ and $\Delta f_{\text {res }}$ values of $\pm 4.6 \%$ and $\pm 1.7 \%$ respectively, to maintain the percentage overlap between bands at $\approx 40 \%$. Reductions in $\Delta K_{V C O}$ and $\Delta f_{r e s}$ are by factors of 6 and 17 respectively over a conventional sub-banded VCO designed using the same process, to meet the same tuning range. This makes the proposed VCO more suited to stable PLL
TABLE II

Performance Comparison Against ReCEnt Publications

\begin{tabular}{|c|c|c|c|c|c|}
\hline Ref. & [4] & {$[5]$} & [6] & [7] & $\begin{array}{l}\text { This } \\
\text { Work }\end{array}$ \\
\hline$\Delta K_{V C O}( \pm \%)$ & 12 & 4.4 & 20.4 & 12.5 & 4.6 \\
\hline$\Delta f_{\text {res }}( \pm \%)$ & $\mathrm{N} / \mathrm{A}^{a}$ & $\mathrm{~N} / \mathrm{A}^{a}$ & $\mathrm{~N} / \mathrm{A}^{a}$ & 9 & 2.2 \\
\hline$\Delta F(\mathrm{MHz})$ & 1320 & 1050 & 720 & 825 & 1150 \\
\hline Technology & $\begin{array}{c}0.25 \mu \mathrm{m} \\
\mathrm{BiCMOS}\end{array}$ & $\begin{array}{l}0.13 \mu \mathrm{m} \\
\mathrm{CMOS}\end{array}$ & $\begin{array}{l}0.18 \mu \mathrm{m} \\
\text { CMOS }\end{array}$ & $\begin{array}{l}0.18 \mu \mathrm{m} \\
\text { CMOS }\end{array}$ & $\begin{array}{c}\text { 90nm } \\
\text { CMOS }\end{array}$ \\
\hline $\begin{array}{c}\text { Power } \\
\text { Supply (V) }\end{array}$ & 2.8 & 2.8 & 1.8 & 1.8 & 1 \\
\hline Power $(\mathrm{mW})$ & 11.2 & $-b$ & 9 & $-b$ & 0.85 \\
\hline
\end{tabular}

${ }^{a} \Delta f_{\text {res }}$ not addressed, hence reducing PVT robustness.

${ }^{b}$ Not specified for VCO used.

operation. In addition it reduces $K_{V C O}$ requirements resulting in a phase noise improvement over the conventional VCO by $2 \mathrm{~dB}$. Reduced loading of $Q_{\text {tank }}$ then keeps the overall power consumption extremely low at $850 \mu \mathrm{W}$ from a $1 \mathrm{~V}$ supply.

\section{ACKNOWLEDGMENT}

The authors would like to thank Enterprise Ireland (EI) and the Microelectronics Competence Centre of Ireland (MCCI) for funding of this research.

\section{REFERENCES}

[1] B. Razavi, "A Study of Phase Noise in CMOS Oscillators," IEEE Journal of Solid-State Circuits, vol. 31, pp. 331 - 343, Mar. 1996.

[2] A.Kral, F. Behbahani, and A. Abidi, "RF-CMOS Oscillators with Switched Tuning," IEEE Custom Integrated Circuits Conference, pp. 555 - 558, 1998.

[3] D. Hauspie, E. Park, and J. Craninckx, "Wideband VCO with Simulataneous Switching of Frequency Band, Active Core, and Varactor Size," IEEE Journal of Solid-State Circuits, vol. 42, pp. 1472 - 1480, July 2007.

[4] T. Nakamura et al., "A Wide-tuning-range VCO with Small VCOgain Fluctuation for Multi-band W-CDMA RFIC," Proceedings of the European Solid State Circuits Conference, pp. 448 - 451, Sept. 2006.

[5] P. Chen et al., "A $0.13 \mu \mathrm{m}$ CMOS Quad-Band GSM/GPRS/EDGE RF Transceiver Using a Low-Noise Fractional-N Frequency Synthesizer and Direct-Conversion Architecture," IEEE Journal of Solid-State Circuits, vol. 44, pp. 1454 - 1463, May 2009.

[6] K. Fu et al., "Design of Wideband LC VCO with small KVCO fluctuation for RFID Synthesizer Application," Proc. of Intl. Conference on Communications Technology, pp. 286 - 289, Dec. 2008.

[7] L. Lu et al., "An 18-mw 1.175-2-GHz Frequency Synthesizer With Constant Bandwidth for DVB-T Tuners," IEEE Transactions on Microwave Theory and Techniques, vol. 57, pp. 928 - 937, Apr. 2009.

[8] M. Tiebot, "Low-Power Low-Phase-Noise Differentially Tuned Quadrature VCO Design in Standard CMOS," IEEE Journal of Solid-State Circuits, vol. 36, pp. 1018 - 1024, July 2001.

[9] A. Zanchi, C. Samori, S. Levantino, and A. Lacaita, "A 2-V 2.5-GHz$104-\mathrm{dBc} / \mathrm{Hz}$ at $100 \mathrm{kHz}$ Fully Integrated VCO with Wide-Band LowNoise Automatic Amplitude Control Loop," IEEE Journal of Solid-State Circuits, vol. 36, pp. 611 - 619, Apr. 2001.

[10] S. Levantino et al., "Frequency Dependence on Bias Current in $5-\mathrm{GHz}$ CMOS VCOs: Impact on Tuning Range and Flicker Noise Upconversion," IEEE Journal of Solid-State Circuits, vol. 37, pp. 1003 - 1011, Aug. 2002.

[11] A. Momtaz et al., "A Fully Integrated SONET OC-48 Transceiver in Standard CMOS," IEEE Journal of Solid-State Circuits, vol. 36, pp. 1964 - 1973, Dec. 2001.

[12] S. Lee et al., "A Quad-Band GSM-GPRS Transmitter With Digital AutoCalibration," IEEE Journal of Solid-State Circuits, vol. 39, pp. 2200 2214, Dec. 2004. 